\title{
Atypical Williams Syndrome in a Child who Presented with Aortic Stenosis and Coarctation and a Positive Family History
}

Ashleigh Hansen, BSc, MSc, LCGC, CCGC, Lily Radanovich, BA, Robin Dawn Clark, MD

\section{Case Summary:}

A 6-day old male was referred for a genetics consultation for prenatally diagnosed aortic stenosis and a positive maternal history of aortic stenosis. He was born at 37 weeks 6 days gestation to a 34-year-old G4SAb3 mother with chronic hypertension and superimposed preeclampsia. A fetal echocardiogram at 33 weeks 6 days gestation showed moderate left ventricular hypertrophy, normal aortic root, mild mitral regurgitation, possible bicuspid aortic valve, and mild aortic stenosis. His birth weight was $3.51 \mathrm{~kg}$ with APGAR scores of $8^{1}$ and $8^{5}$.

"His physical exam was notable for mild periorbital puffiness, micrognathia, thin upper lip, long philtrum, milia on the nose, and dimples on both knees (Figure 1). His palate was high arched. He had mild tachypnea, subcostal retractions, and perioral cyanosis."

His physical exam was notable for mild periorbital puffiness, micrognathia, thin upper lip, long philtrum, milia on the nose, and dimples on both knees (Figure 1). His palate was high arched. He had mild tachypnea, subcostal retractions, and perioral cyanosis. At one day of age, an echocardiogram showed a diffusely small aortic arch with an isthmus of $2.1 \mathrm{~mm}$, transverse arch of $3 \mathrm{~mm}$, and ascending aorta of $6 \mathrm{~mm}$. There was mild coarctation with a peak velocity of $2.7 \mathrm{~m} / \mathrm{s}$. The aortic valve was mildly hypoplastic and bicuspid with a fusion of the right and non-coronary cusps. The calcium value was normal. Chromosome microarray revealed 2 deletions: one within and one near to the Williams syndrome

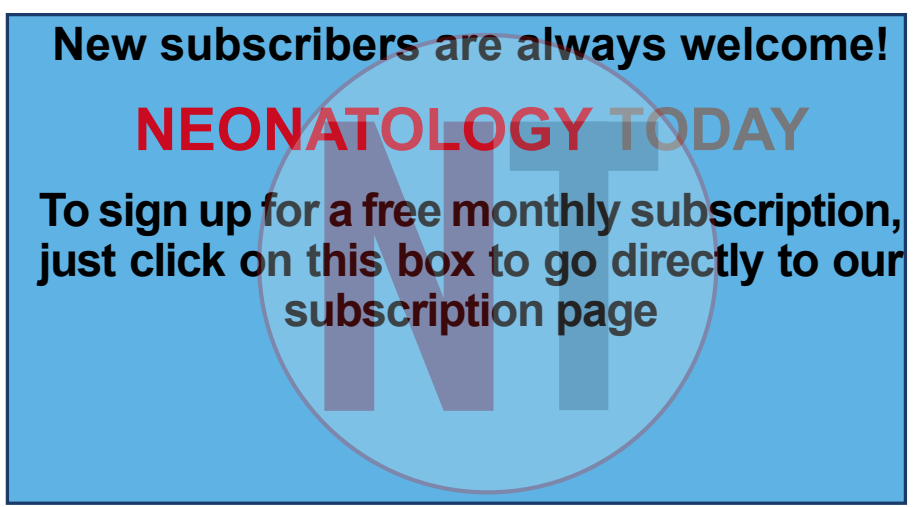

critical region on chromosome 7q: arr[hg19] 7q11.23(73,156,045$73,810,341) \times 1,7 q 11.23(74,350,950-75,294,796) \times 1$.

He underwent aortic arch reconstruction with resection of aortic isthmus and coarctation at 23 days of life. Post-surgical echocardiogram at 29 days of age showed no residual coarctation of the aorta but a $4 \mathrm{~mm}$ hypoplastic descending aorta. There was moderate supravalvar aortic stenosis, narrowing to $4.3 \mathrm{~mm}$ with an increased velocity of $3.7 \mathrm{~m} / \mathrm{s}$. There was severe right and moderate left branch pulmonary artery stenosis, moderate hypertrophy of the left and right ventricles. Moderate mid-cavitary dynamic obstruction was noted.

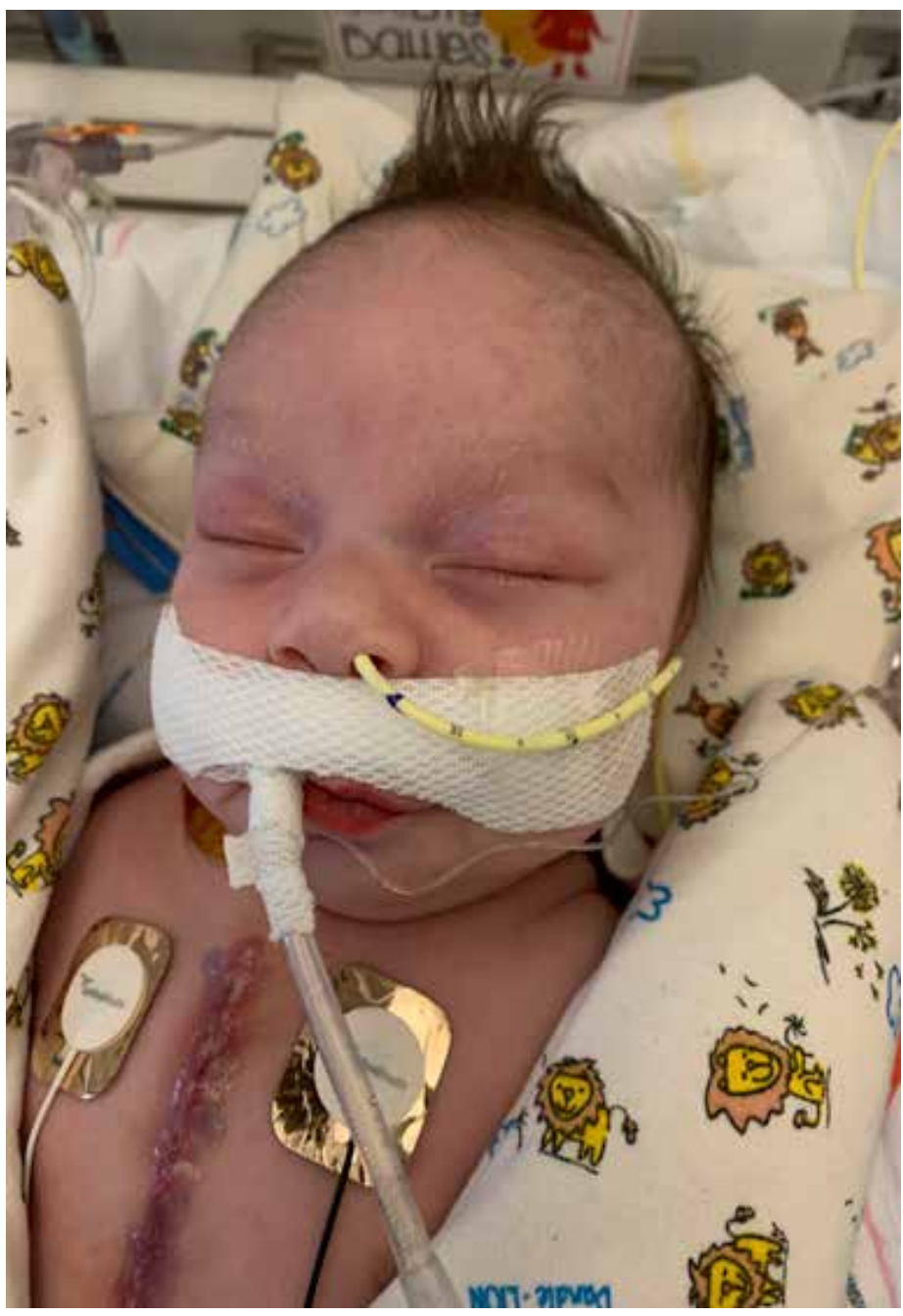

Figure 1 The patient's facial features at about 25 days of age are partially obscured by tape. He has a depressed nasal bridge and mild micrognathia, but he lacks other facial features of Williams syndrome, including thick lips and an open mouth. 


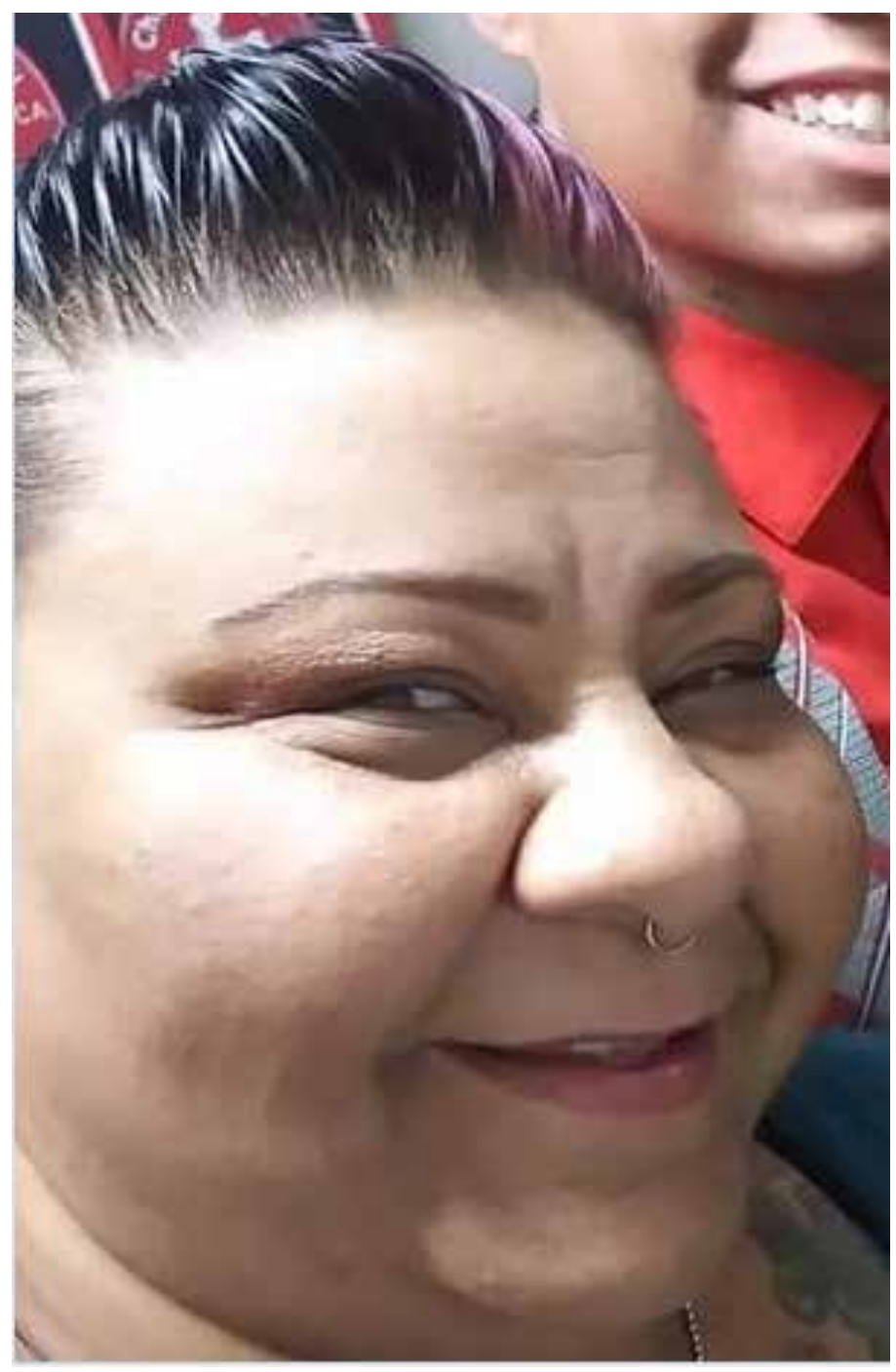

Figure 2 The patient's mother has upslanting palpebral fissures, a bulbous nasal tip, and a thin upper lip. However, she lacks many typical facial features of Williams syndrome, including anteverted nares, patulous, prominent lips, and enamel hypoplasia.

\section{Family History:}

The patient's mother, a 34-year-old woman of African American and Caucasian descent, is an articulate and competent historian. She is obese and chronically hypertensive (Figure 2). She has aortic stenosis that has not required surgery. Her echocardiogram during this gestation revealed moderate concentric left ventricular hypertrophy, normal-sized aortic root, mild mitral regurgitation, possibly bicuspid aortic valve, elevated velocity across descending aorta at $2.5 \mathrm{~m} / \mathrm{sec}$, no clear Doppler signal of coarctation, and mild aortic stenosis. She reported mild learning disabilities and self-diagnosed ADHD. She denied hyperacusis and reported that she has good drawing skills. She does not have an overly friendly or chatty personality.
The patient's mother has had three previous spontaneous abortions. Her conventional metaphase chromosome analysis was normal. The patient's father is a 29-year-old male of African American and Caucasian descent. Consanguinity was denied. No one else in the family has congenital cardiac anomalies.

\section{Discussion:}

This child and his mother have aortic stenosis/hypoplasia that has been transmitted in an autosomal dominant pattern (OMIM 185500). (1) Microarray testing in the infant revealed two deletions at $7 q 11.23$, in and near the region associated with Williams syndrome (WS), highlighted on the gene map in Figure 3. Targeted FISH studies for both of these deletions are in progress for the mother.

The smaller, pathogenic deletion at 7q11.23 (654 kb) encompasses 12 genes within the Williams syndrome critical region from CLDN3 to CLIP2, including ELN, the gene that encodes elastin (OMIM 130160). Elastin is a key component in arterial development. Lack of elastin causes obstructive arterial disease by increasing subendothelial production of smooth muscle. Deletion of ELN causes aortic hypoplasia, one of the characteristic features of Williams syndrome. (1) Heterozygous loss of ELN can also affect the renal, mesenteric, and coronary arteries leading to hypertension, abdominal pain, cardiac hypoperfusion with ST-elevation, or sudden death. (2) Elastin deficiency explains the cardiac anomalies in both mother and child and likely contributes to the mother's hypertension. The more distal and larger deletion at 7q11.23 (944 $\mathrm{kb}$ ) is of uncertain significance. It lies outside the region associated with Williams syndrome. This deletion encompasses 33 genes from RCC1L to HIP1.

\section{"The more distal and larger deletion} at $7 q 11.23(944 \mathrm{~kb})$ is of uncertain significance. It lies outside the region associated with Williams syndrome. This deletion encompasses 33 genes from RCC1L to HIP1."

The classic deletion associated with Williams syndrome (WS) is $1.55-1.8 \mathrm{Mb}$ in size, spanning $26-28$ genes, on chromosome 7q11.23. (1) Atypical deletions account for $2-5 \%$ of reported cases. (3) Our patient's pathogenic deletion is nested within this region, but it is much smaller. Recurrent deletions are the result of non-allelic homologous recombination events within chromosome pairs during meiosis. Unequal exchanges between homologous chromosomes in a chromosome pair cause de novo copy number variation, (2) both deletions and duplications. Regions of DNA with a high density of repetitive elements, such as chromosome $7 q 11.23$, are more susceptible to unequal recombination events. See Figure 3 for a map of low copy repeats in this region of chromosome 7q. Our patient's deletions, which are discontinuous and are not bordered by low copy repeats, may have a different etiology.

The only worldwide monthly publication
exclusively serving Pediatric and Adult
Cardiologists that focus on Congenital/
Structural Heart Disease (CHD), and
CONGENITAL
CARDLOGY
TODAY




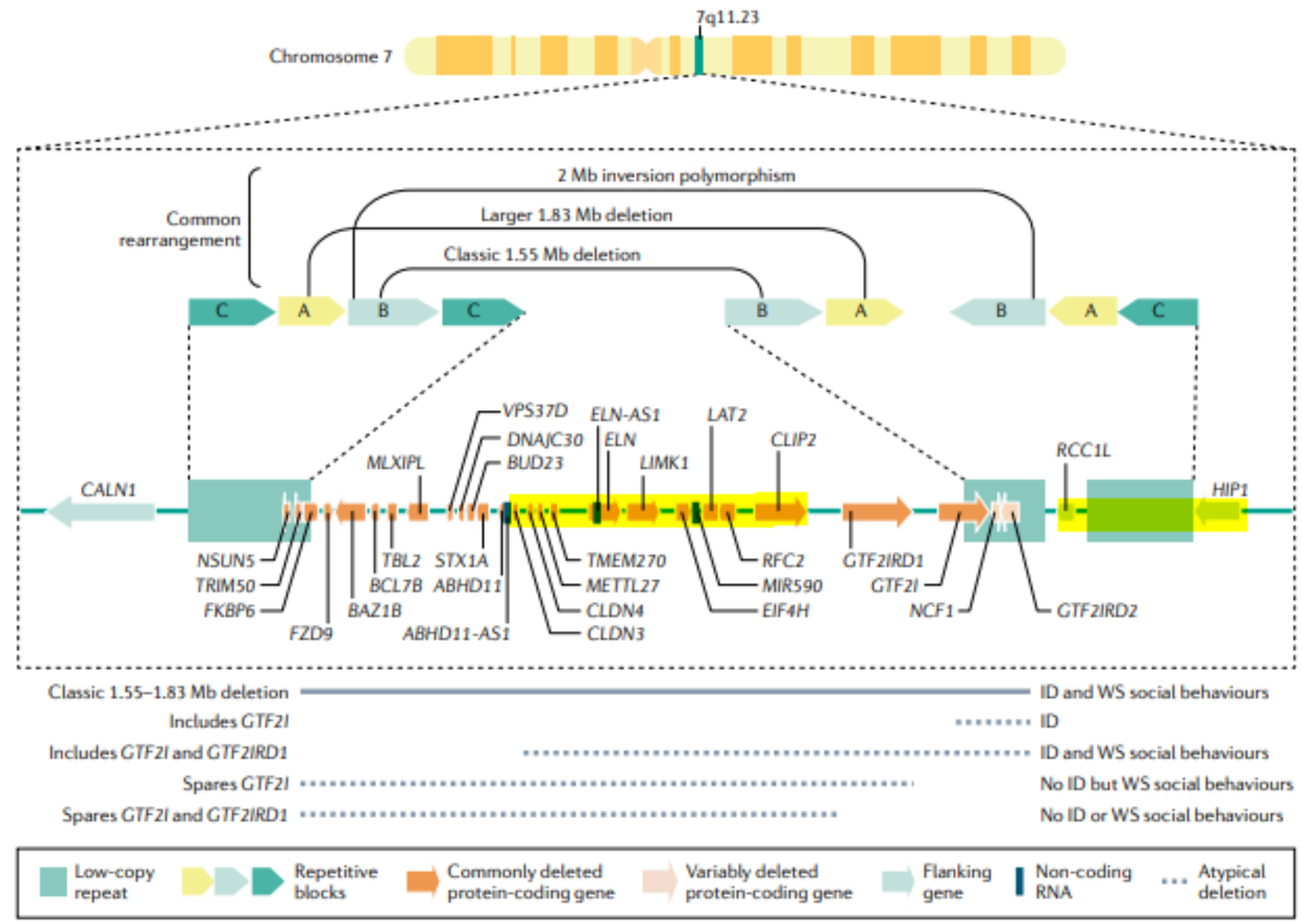

Figure 3: (adapted from Kozel et al. 2021). (1) The regions highlighted in yellow are deleted in this patient. The smaller deletion is within the Williams Syndrome Critical Region, and it includes ELN, the gene that encodes elastin. The patient's deletion does not include GTFR2IRD1 and GTF2I (the unhighlighted region between the two deletions), which have been implicated in the etiology of intellectual disability in individuals with classic Williams syndrome.

\section{"It is unclear if our patient will express the neurocognitive and behavioral phenotypes associated with WS, but there is reason to expect that he will not. Evidence suggests that GTF2IRDI, GTF2IRD2, GTF2I have}

\section{important roles in developing cognitive and visual-spatial abilities."}

Our patient's smaller deletion explains why he does not have the classic phenotype associated with WS. Specifically, he lacks the expected facial features and hypercalcemia. Individuals with WS exhibit a combination of high sociability and empathy for others with high anxiety. They exhibit relative strengths in socialization and communication skills and weakness in daily living skills. Indi- viduals with WS typical have hyperacusis, visual-spatial disabilities (inability to draw), a "cocktail party" personality, and intellectual disability.

It is unclear if our patient will express the neurocognitive and behavioral phenotypes associated with WS, but there is reason to expect that he will not. Evidence suggests that GTF2IRDI, GTF2IRD2, GTF2I have important roles in developing cognitive and visual-spatial abilities. Battista Ferrero et al. reported a male child with mild WS physical features, average intelligence with normal $I Q$, and only some features of the expected neuropsychological WS profile. (4) He carried a smaller atypical deletion of $\sim 1 \mathrm{Mb}$ that did not include GTF2IRD1 and GTF2I and only partially included $B A Z 1 B$. Their report supports the hypothesis that GTF2IRD1 and GTF2I hemizygosity are associated with WS-specific motor and cognitive deficits. Because of this, we are cautiously optimistic that our patient may be spared intellectual disability because his deletion does not include GTFR2IRD1 and GTF2I. However, other genes that are deleted in our patient may affect his neurocognitive ability. (2) The loss of LIMK1 is associated with visual processing defects and other neurocognitive aspects of the WS phenotype including anxiety, depression, and attention deficit. (5) The typical craniofacial features of WS are also absent in our pa- 
tient, probably due to the preservation of GTF2IRDI. Alesi et al. suggest that lack of CLIP2, GTF2IRD1, and GTF2I are associated with the visual-spatial deficit, cognitive and behavioral profile of WS, and they implicate haploinsufficiency for GTF2IRD1 in the facial dysmorphology. (6) Deletion of GTF2IRD2 may play a role in the dental anomalies seen in WS, such as hypoplastic enamel. (7) Preservation of NCF1 may pose an increased risk for hypertension in this patient. Individuals with WS who have a deletion of NCF1 have a decreased risk for hypertension and have protection from vascular stiffness. $(8,9)$ Our patient may also be spared from hypercalcemia as this has been linked to a loss of $B A Z 1 B$, which is retained in our patient. $(4,10)$

The second, larger, and more distal $944 \mathrm{~kb}$ deletion is considered a variant of uncertain significance. However, some patients with an overlapping deletion have had an intellectual disability, epilepsy, and behavioral problems. Deletions distal to the WS critical region that include HIP1 and YWHAG have been associated with autistic traits, epilepsy, and intellectual disability $(10,11)$ and may also be associated with inattention, hyperactivity, impulsivity, and aggression. (11)

"The second, larger, and more distal 944 $\mathrm{kb}$ deletion is considered a variant of uncertain significance. However, some patients with an overlapping deletion have had an intellectual disability, epilepsy, and behavioral problems."

\section{Practical Applications:}

1. Order a chromosome microarray in infants with aortic stenosis, even when they do not have the typical facial features or other characteristics of a syndrome such as Williams syndrome. Recall that atypical deletions cause $2-5 \%$ of Williams syndrome.

2. Test parents for microarray abnormalities identified in their child. In this case, genetic testing in the mother, who also has aortic stenosis, may inform this child's prognosis for intellectual and learning abilities.

3. Take a careful family history whenever an infant has a congenital anomaly. The diagnosis of atypical Williams syndrome in this patient may lead to the same diagnosis in his mother, which could affect the management of her cardiac defect and chronic hypertension.

4. In children and adults with congenital heart defects, atypical deletions in the Williams syndrome critical region are likely to be underdiagnosed in part due to the absence of other expected features.

\section{References:}

1. Morris CA. Williams Syndrome. 1999 Apr 9 [Updated 2017 Mar 23]. In: Adam MP, Ardinger HH, Pagon RA, et al., editors. GeneReviews ${ }^{\circledR}$ [Internet]. Seattle (WA): Univ. of Washington, Seattle; 1993-2021. https://www.ncbi.nlm.nih. gov/books/NBK1249/. PMID: 20301427

2. Kozel BA, Barak B, Kim CA, et al. Williams syndrome. Nat Rev Dis Primers. 2021 Jun 17;7(1):42. PMID: 34140529.

3. Lugo M, et al. Social, neurodevelopmental, endocrine, and head size differences associated with atypical deletions in Williams-Beuren syndrome. Am J Med Genet A. 2020 May;182(5):1008-1020. PMID 32077592.

4. Battista Ferrero, G., Howald, C., Micale, L. et al. An atypical $7 q 11.23$ deletion in a normal IQ Williams-Beuren syndrome patient. Eur J Hum Genet 18, 33-38 (2010). https://doi-org. uml.idm.oclc.org/10.1038/ejhg.2009.108 PMID: 19568270.

5. Streată I, Serban-Soșoi S, Budișteanu M, et al. Array CGH - A Powerful Tool in Molecular Diagnostic of Pathogenic Microdeletions - Williams-Beuren Syndrome - A Case Report. Curr Health Sci J. 2016 Apr-Jun;42(2):207-212. PMID: 30568834.

6. Alesi V, Loddo S, Orlando V, et al. Atypical 7q11.23 deletions excluding ELN gene result in Williams-Beuren syndrome craniofacial features and neurocognitive profile. Am J Med Genet A. 2021 Jan;185(1):242-249. PMID: 33098373.

7. Ohazama A, Sharpe PT. TFIl-I gene family during tooth development: candidate genes for tooth anomalies in Williams syndrome. Dev Dyn. 2007 Oct;236(10):28848. PMID: 17823943.

8. Del Campo M, Antonell A, Magano LF, et al. Hemizygosity at the NCF1 gene in patients with Williams-Beuren syndrome decreases their risk of hypertension. Am J Hum Genet. 2006;78:533-42. PMID: 16532385.

9. Kozel BA, Danback JR, Waxler JL, et al. Williams syndrome predisposes to vascular stiffness modified by antihypertensive use and copy number changes in NCF1. Hypertension. 2014 Jan;63(1):74-9. PMID: 24126171.

10. Fusco C, Micale L, Augello B, et al. Smaller and larger deletions of the Williams Beuren syndrome region implicate genes involved in mild facial phenotype, epilepsy and autistic traits. Eur J Hum Genet. 2014 Jan;22(1):64-70. PMID: 23756441

11. Ramocki MB, Bartnik M, Szafranski $P$, et al. Recurrent distal 7q11.23 deletion including HIP1 and YWHAG identified in patients with intellectual disabilities, epilepsy, and neurobehavioral problems. Am J Hum Genet. 2010 Dec 10;87(6):857-65. PMID: 21109226.

Disclosures: The authors have no relevant disclosures.

\section{NT}

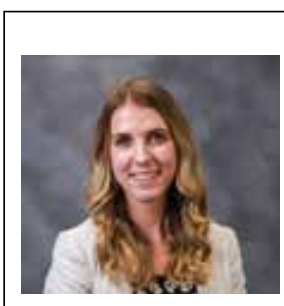

Ashleigh Hansen, BSC, MSc, LCGC, CCGC

Pediatric Genetic Counselor

Loma Linda University School of Medicine

Division of Genetics

Department of Pediatrics

Email: ahansen@llu.edu 